\title{
Pediatric composite nodal marginal zone lymphoma and classical Hodgkin lymphoma
}

\author{
Shanxiang Zhang $\cdot$ Kristin Post $\cdot$ Liang Cheng
}

Received: 30 May 2014 / Accepted: 31 July 2014 / Published online: 30 August 2014

(C) Springer-Verlag Berlin Heidelberg 2014

\section{Introduction}

Composite lymphoma is defined as different types of lymphoma occurring simultaneously in the same tissue or mass [1,2]. Composite classical Hodgkin lymphoma (CHL) and nonHodgkin lymphoma (NHL) are uncommon. The reported NHLs in composite lymphoma are most commonly B cell lymphoma, including diffuse large B cell lymphoma, follicular lymphoma, mantle cell lymphoma, marginal zone lymphoma (MZL), and chronic lymphocytic leukemia/small lymphocytic lymphoma [3-8]. Composite lymphoma with CHL and MZL is rare and has never been reported in pediatric population [9-13]. Here, we report a composite lymphoma composed of nodal MZL and CHL in a previously healthy teenage boy.

\section{Case history}

A 15-year-old previously healthy boy initially presented with a left supraclavicular mass. An ultrasound-guided needle core biopsy performed at an outside institution was reported as chronic lymphadenitis with immunophenotypically unremarkable T cells and polyclonal B cells detected by concurrent flow cytometric analysis (FCA). No treatment was offered. The mass subsequently grew. An excisional biopsy of the enlarged left cervical lymph node performed 3 months after the initial biopsy showed reactive follicular hyperplasia. During this period, the patient started to develop fever, body aches, decreased appetite, and body weight loss of 10 to $15 \mathrm{lb}$.

S. Zhang $(\bowtie) \cdot K$. Post $\cdot$ L. Cheng

Department of Pathology and Laboratory Medicine, Indiana

University, 350 West 11th Street, Room 5042, Indianapolis,

IN 46202, USA

e-mail: sz5@iupui.edu
The patient was referred to our institute and a computed tomographic scan showed significantly enlarged left cervical, supraclavicular, axillary, and superior mediastinal lymph nodes. There was no disease in the orbits, sinuses, nasal cavity, pharynx, oral cavity, hypopharynx and larynx, thyroid, salivary glands, abdomen, liver, or spleen. An excisional biopsy of the left supraclavicular lymph node was performed in our institute approximately 5 months after the initial needle biopsy. A diagnosis of composite lymphoma was rendered. The previous two biopsies were also reviewed in our institute. While confirming the diagnosis of the second biopsy, a diagnosis of atypical lymphoid proliferation concerning for Hodgkin lymphoma was rendered for the first biopsy. A staging bone marrow biopsy showed no evidence of lymphoma. The patient was treated with ABVE-PC per protocol AHOD0031 (doxorubicin hydrochloride, bleomycin, vincristine sulfate, etoposide, prednisone, cyclophosphamide) for classical Hodgkin lymphoma followed by radiation therapy $(2,100 \mathrm{cGy})$. The patient has been in remission for 8 months at the time of last follow-up.

\section{Materials and methods}

Clinical information and the results of FCA were collected from the medical records. Immunohistochemical stains were performed using the prediluted, ready-to-use antibodies (CD5, 4C7; CD10, 56C6; CD15, Carb-3: CD20, L26; CD30, Ber-H2; CD43, DF-T1; CD45/RB, 2B11+PD7/26; CD138, MI15; cyclin D1, EP12; IgD, code TR517; kappa light chain, code IR506; lambda light chain, code IR507; and Ki-67, MIB-1) from Dako (Carpinteria, CA), anti-LEF1 (clone: EPR2029Y, 1:100, Abcam Inc., Cambridge, MA) with a Dako Autostainer Plus instrument following the manufacturer's protocols. 


\section{Results}

The excised mass measuring $6.5 \times 5.3 \times 2.5 \mathrm{~cm}$ was divided for FCA and histologic evaluation. FCA revealed a distinct monoclonal B cell population with expression of CD19, CD20, CD22, CD5 (partial), and surface kappa light chain. The monoclonal B cells were negative for CD10 and FMC-7 (Fig. 1). The expression of CD23 was equivocal due to suboptimal stain. The histologic sections showed near complete effacement of the nodal architecture by a proliferation of small atypical lymphoid cells (Fig. 2a, b) adjacent to areas with a nodular cellular proliferation divided by thick fibrosing bands (Fig. 3a). The small atypical lymphoid cells were positive by immunohistochemical stains (IHC) for CD20 and negative for CD5, LEF-1, CD10, CD43, cyclin D1, and IgD. CD21 stain revealed focally expanded and disrupted follicular dendritic cell meshworks. Ki-67 stain highlighted residual germinal centers and relatively high proliferation in the marginal zone, imparting a targetoid pattern (Fig. 2c-f). There were scattered polyclonal plasma cells as shown by IHC for CD138 and kappa and lambda light chains. The morphology and the presence of monoclonal B cell population without expression of CD5 (by IHC), CD10, cyclin D1, and LEF-1 support the diagnosis of marginal zone lymphoma over a small lymphocytic lymphoma and other types of B cell lymphoma. The nodular areas comprised of scattered, large atypical, mostly mononuclear, rarely binuclear, and multinuclear cells with prominent eosinophilic nucleoli and abundant clear cytoplasm in the background of small lymphocytes, histiocytes, plasma cells, and eosinophils (Fig. 3b). The large atypical cells were weakly positive for PAX-5 (Fig. 3c) and positive for CD30 (Fig. 3d) and CD15 (Fig. 3e). They were negative for CD45RB, CD20, OCT.2, and BOB.1 (Fig. 3f). In situ hybridization for Epstein-Barr virus-encoded RNAs (EBER) was positive in the large atypical cells (Fig. $3 \mathrm{~g}$ ).

\section{Discussion}

Pediatric nodal MZL is a rare entity with distinctive clinical and morphological features. It typically occurs in young males and presents as an asymptomatic, localized cervical lymphadenopathy (stage I). Disruption of residual follicles morphologically resembling progressive transformation of germinal centers is seen in approximately twothirds of the cases. Majority of the reported cases were shown to be clonal by molecular study for B cell receptor (BCR) gene rearrangement, though 5-10\% of cases showed the presence of clonal B cell population only by immunophenotyping. The prognosis for pediatric nodal MZL is excellent even without any systemic treatment $[12,14,15]$. A close differential diagnosis is atypical marginal zone hyperplasia with monotypic immunoglobulin expression (aMZH). To date, aMZH has only been reported in mucosa-associated lymphoid tissue with a monotypic lambda chain expression [16, 17]. The MZL
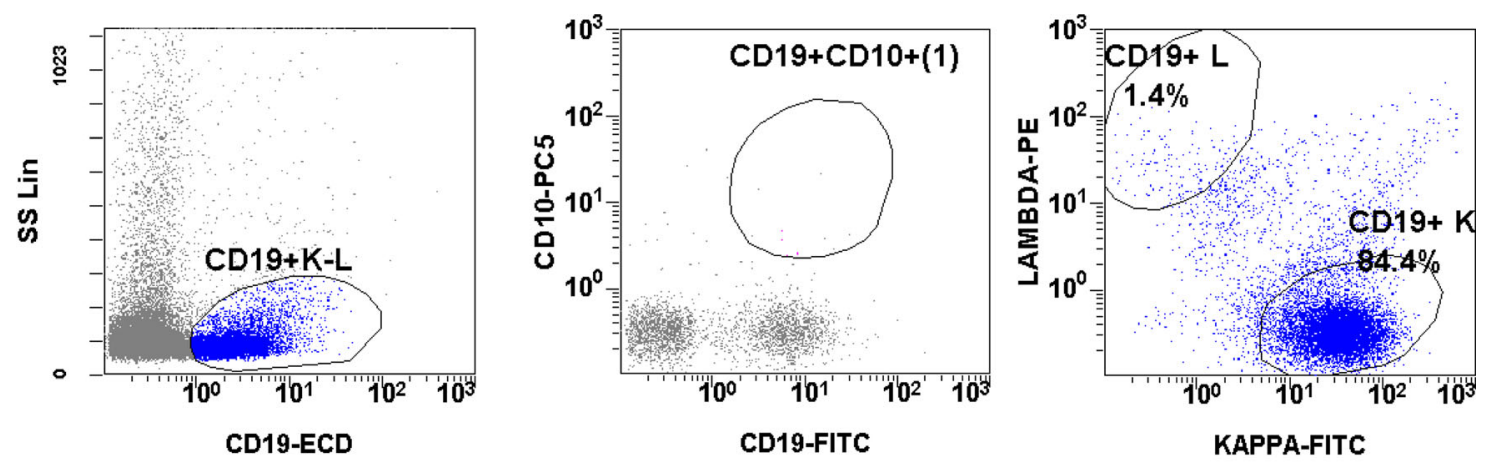

OT ( debris ) AND TIME GT.] FL4 Log/FL3
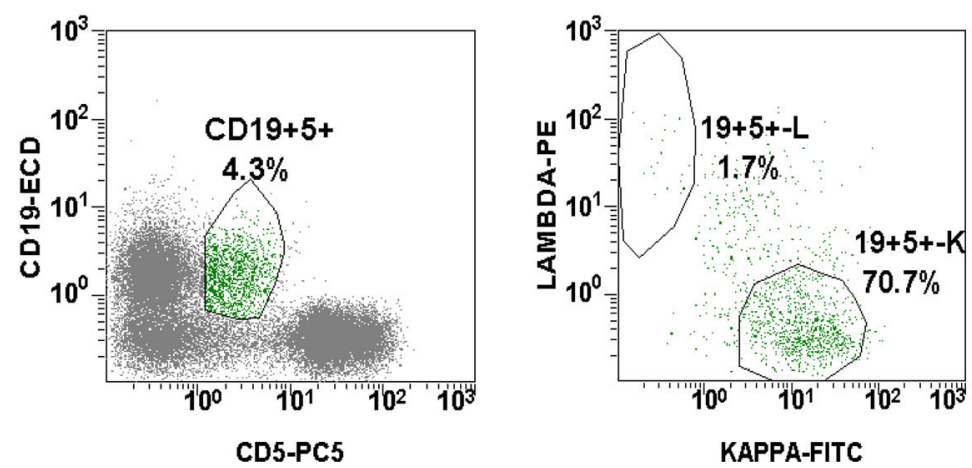

Fig. 1 A distinct kappa-restricted CD5+ (partial), CD10- monoclonal B cell population detected by flow cytometric analysis 
Fig. 2 Morphologic and immunophenotypical features of marginal zone lymphoma component. a-b Hematoxylin and eosin, $\mathbf{a} \times 40$ and $\mathbf{b} \times 400$. $\mathbf{c}-\mathbf{g}$ Immunohistochemistry, $\times 100$ : c CD20, d CD5, e LEF-1, f CD21, and $\mathbf{g ~ K i}-67$
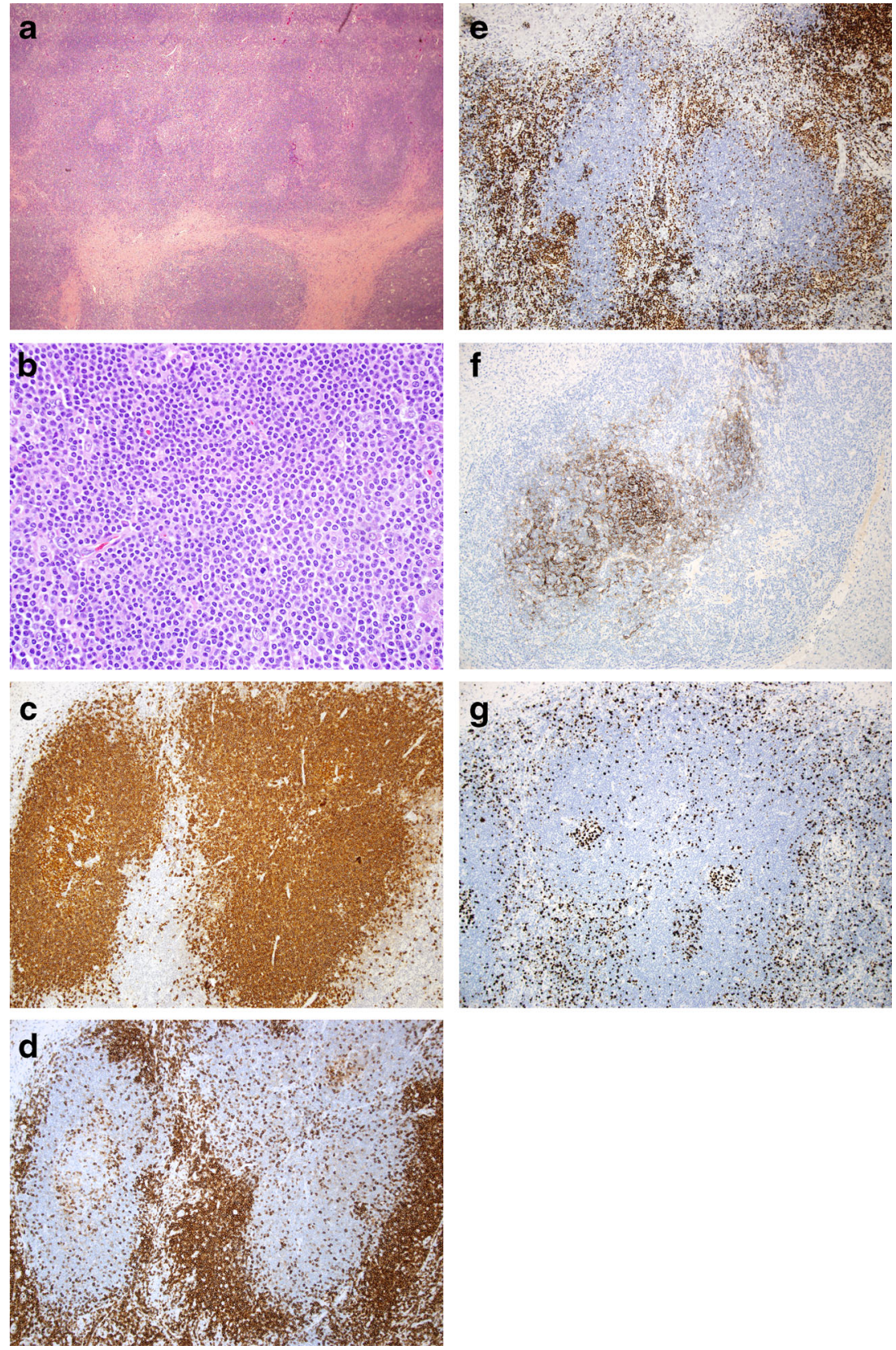

in the current case involved supraclavicular lymph node and showed a kappa chain restriction by FCA. Though the molecular study for BCR gene rearrangement failed to detect a clonal BCR rearrangement (data not shown), the clinical presentation and kappa chain restriction in the $\mathrm{B}$ cell population supports the diagnosis of MZL over aMZH.

Composite CHL and MZL have been rarely reported in adults and elder population. They most commonly involve extranodal tissues, such as the stomach, lung, and thyroid [9-11, 13]. CHL has been proposed, in portion of the cases, as a progression of MZL, particularly in composite CHL and extranodal MZL of mucosa-associated lymphoid tissue (MALT lymphoma). The involved MALT lymphomas tended to be $\mathrm{t}(11 ; 18)(\mathrm{q} 21 ; \mathrm{q} 21)$ negative. EBV infection may pathogenically contribute to this progression $[13,18,19]$. In this report, we described a unique case of composite CHL and nodal MZL in a previously healthy child. EBV was detected in the CHL component but not in MZL. As there was suspicion of $\mathrm{CHL}$ at the time of first biopsy, the CHL less likely developed as a consequence of progression of the MZL. Could this case represent an exceedingly rare event of CHL and MZL developing independently by chance or there is some unknown factor which may contribute to the development of both CHL and MZL? Additional studies including cytogenetic analysis, array comparative genomic hybridization, and whole exome sequencing may provide some insight 
Fig. 3 Morphologic and immunophenotypical features of classical Hodgkin lymphoma component. a-b Hematoxylin and eosin, $\mathbf{a} \times 100$ and $\mathbf{b} \times 500$. $\mathbf{c}-\mathbf{f}$ Immunohistochemistry, $\times 200$ : c PAX-5, d CD30, e CD15, and f OCT.2. $\mathbf{g}$ In situ hybridization for Epstein-Barr virus-encoded RNAs
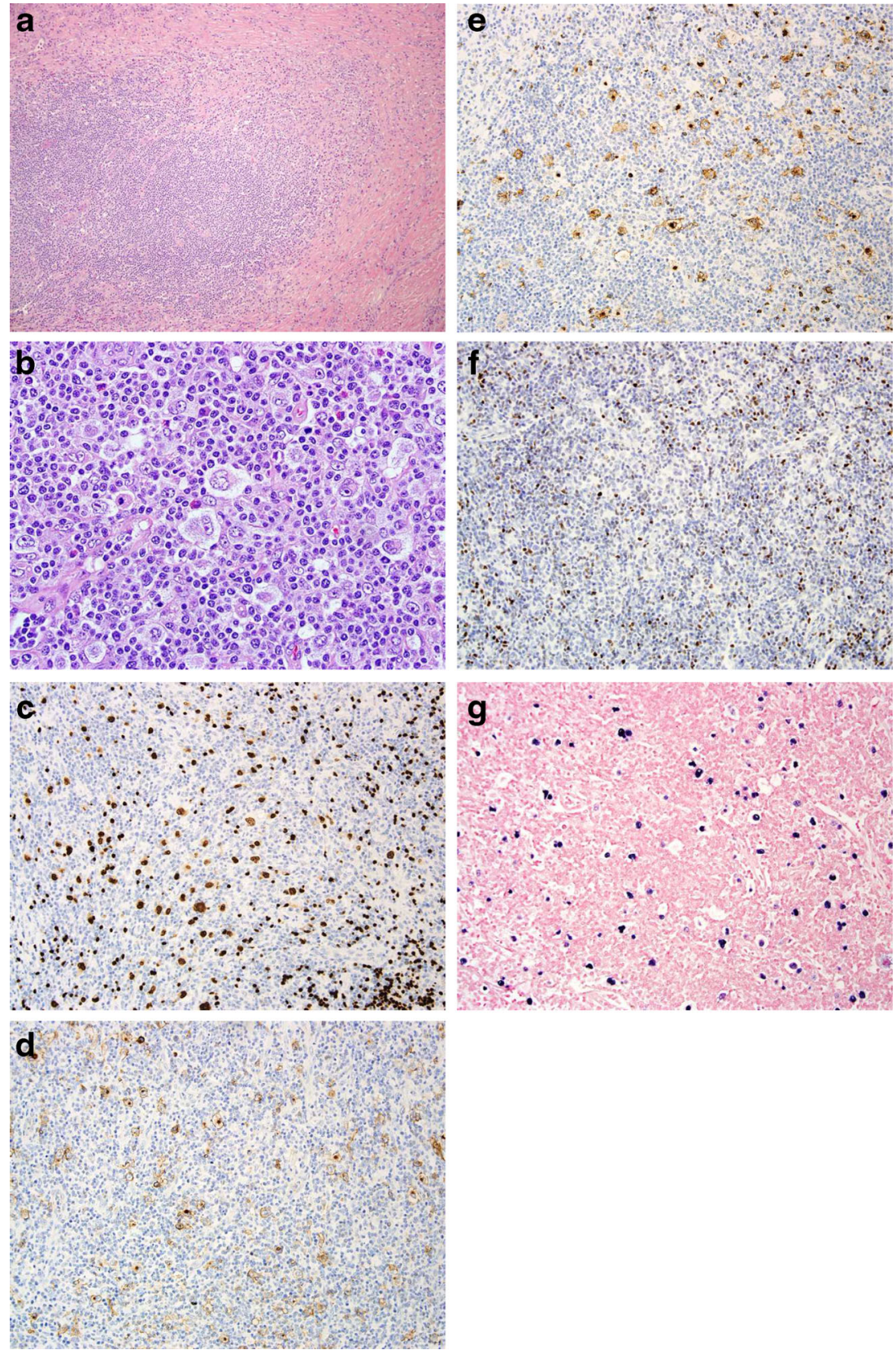

biopsy specimen. The coexistence of both benign and neoplastic lymph nodes in the same anatomic site is suggestive of some common factors contributing to the development of lymphoid hyperplasia, MZL, and CHL.

In summary, this is the first report, to the best of our knowledge, of a pediatric composite CHL and MZL. The findings of reactive follicular hyperplasia, CHL, and MZL in the contiguous lymph nodes before any treatment indicate that they may share some common pathogenic pathways. This unusual case also stresses the importance of adequate sampling in the workup of lymphoma, especially when there is a discrepancy between the tissue diagnosis and clinical presentation. 


\section{References}

1. Hicks EB, Rappaport H, Winter WJ (1956) Follicular lymphoma; a re-evaluation of its position in the scheme of malignant lymphoma, based on a survey of 253 cases. Cancer 9(4):792-821

2. Kim H, Hendrickson R, Dorfman RF (1977) Composite lymphoma. Cancer 40(3):959-976

3. Kim H (1993) Composite lymphoma and related disorders. Am J Clin Pathol 99(4):445-451

4. Hayes SJ et al (2006) Composite mantle-cell lymphoma and classical Hodgkin lymphoma. Histopathology 48(5):621-623

5. Oka K et al (2010) Coexistence of primary pulmonary Hodgkin lymphoma and gastric MALT lymphoma associated with EpsteinBarr virus infection: a case report. Pathol Int 60(7):520-523

6. Rathnam K et al (2011) Composite Hodgkin lymphoma and chronic lymphocytic leukemia: a rare case. J Cancer Res Ther 7(4):484-485

7. Wang HW et al (2013) Composite diffuse large B-cell lymphoma and classical Hodgkin's lymphoma of the stomach: case report and literature review. World J Gastroenterol 19(37):6304-6309

8. Yu G et al (2011) Composite lymphoma in the anterior mediastinum: a case report and review of the literature. Diagn Pathol 6:60

9. Elmahy H, Hawley I, Beard J (2007) Composite splenic marginal zone lymphoma and classic Hodgkin lymphoma - an unusual combination. Int J Lab Hematol 29(6):461-463

10. Harada $\mathrm{S}$ et al (2008) Classical Hodgkin lymphoma concurrently evolving in a patient with marginal zone B-cell lymphoma of the spleen. Ann Diagn Pathol 12(3):212-216
11. Oka K et al (2012) Concurrent gastric MALT and Hodgkin lymphoma: a case report. Int J Surg Pathol 20(2):201-204

12. Taddesse-Heath L et al (2003) Marginal zone B-cell lymphoma in children and young adults. Am J Surg Pathol 27(4): $522-531$

13. Zettl A et al (2005) Composite marginal zone B-cell lymphoma and classical Hodgkin's lymphoma: a clinicopathological study of 12 cases. Histopathology 46(2):217-228

14. Gitelson E et al (2010) Pediatric nodal marginal zone lymphoma may develop in the adult population. Leuk Lymphoma 51(1):89-94

15. Rizzo KA et al (2010) Marginal zone lymphomas in children and the young adult population; characterization of genetic aberrations by FISH and RT-PCR. Mod Pathol: Off J U S Can Acad Pathol 23(6): $866-873$

16. Attygalle AD et al (2004) Atypical marginal zone hyperplasia of mucosa-associated lymphoid tissue: a reactive condition of childhood showing immunoglobulin lambda light-chain restriction. Blood 104(10):3343-3348

17. Kaur P, Levy NB (2012) Atypical marginal zone hyperplasia of tonsil with immunoglobulin light chain restriction. Am J Hematol 87(4): 424-425

18. Kingma DW et al (1994) Epstein-Barr virus is infrequently identified in non-Hodgkin's lymphomas associated with Hodgkin's disease. Am J Surg Pathol 18(1):48-61

19. Momose $\mathrm{H}$ et al (1992) Chronic lymphocytic leukemia/small lymphocytic lymphoma with Reed-Sternberg-like cells and possible transformation to Hodgkin's disease. Am J Surg Pathol 16(9):859 867 